\title{
LES PROBLĖMES FORESTIERS DE LA SICILE
}

A l'aimable et heureuse initiative de $M$. l'Inspecteur Général Giordano, Professeur à la Faculté Forestière de Florence, le service forestier de Sicile a bien voulu organiser un court voyage d'études auquel ont pris part:

- M. l'Inspecteur Général Giordano,

- MM. Fontaine et Huberman, Chefs de Sections à la Division des Forêts de l'Organisation des Nations Unies pour l'Alimentation et l'Agriculture,

- M. de Vaissière, Conservateur des Eaux et Forêts à la Direction Générale des Eaux et Forêts.

Cette tournée a été dirigée par M. l'Ingénieur PaLtRINIERI, Chef du service forestier de la région de Sicile, avec le précieux concours des Chefs d'Inspection forestière de Catane, Caltanisetta, Enna et Agrigente, le Docteur d'Amato et son adjoint le Dr Piccioni, les Docteurs la Cascia, Griveliz et Morello. La chaleur de l'accueil a rendu encore plus sensible l'intérêt des visites et des observations.

Le voyage a coïncidé avec la fin du Congrès Mondial de l'Eucalyptus les 28 et 29 octobre I956, et s'est prolongé jusqu'au 2 novembre. L'itinéraire choisi a permis de visiter toute la partie orientale et centrale de l'île en passant par Taormine, Catane, Catalgirone, Piazza-Armerina, Vizzini, Siracuse, Modica, Raguse, Gela, Agrigente, Lercara, Palerme.

Les participants ont pu prendre ainsi une connaissance directe des problèmes d'aménagement foncier et de remise en ordre de l'agriculture qui se posent en Sicile, et pour lesquels le service forestier, dans le cadre des activités de la Caisse du Midi, de la Réforme Agraire, de la loi sur la Bonification Intégrale et des lois forestières s'efforce avec compétence et persévérance, de mettre ses techniques au service d'une population qui, dans une proportion de $85 \%$, vit du seul revenu de l'agriculture.

On est frappé par les difficultés de la tâche à entreprendre, mais aussi par l'importance des programmes à l'étude et l'ampleur des travaux en cours. Le Service forestier joue un rôle important et méritoire dans l'effort de tout un peuple pour améliorer les conditions 
d'existence d'un milieu rural dont le niveau de vie est faible, l'évolution démographique en voie de constante augmentation et les possibilités d'évasion vers d'autres emplois limitées.

En terme concret, le gouvernement de Rome et les responsables de la politique en Sicile, ont à faire subsister près de 5 millions d'habitants dont 4 millions vivent directement de l'agriculture sur un territoire de 2500000 hectares.

En dehors des grandes villes situées le plus généralement sur le littoral et qui groupent plus de I million d'âmes dont 600000 dans le principal centre administratif, Palerme, et le surplus dans d'autres chefs-lieux de province comme: Messine, Catane, Siracuse, Enna..., la population se répartit entre les plaines côtières où la densité d'habitat est considérable et le Centre de l'île où elle se concentre dans des agglomérations de 25 à 50000 habitants. I1 s'agit le plus souvent, dans ce dernier cas, de véritables cités dortoirs rurales que les hommes valides quittent chaque jour pour aller travailler la terre, quelquefois à une distance de I 5 ou $20 \mathrm{~km}$. Dans la journée, les rues ne sont animées que par les femmes, les enfants et les vieillards. Les activités commerciales et artisanales sont très peu développées, seuls peuvent être signalés les travaux de broderie dans quelques petites villes, notamment à Mirabelle et à Vizzini, et un renouveau de l'art de la poterie à Caltagirone. Ces conditions de vie et d'habitat agricole extrêmement particulières se rencontrent également dans certaines parties de l'Italie Centrale et dans l'Italie du Sud. Le centre de la Sicile a un relief tourmenté qui rend les communications lentes et difficiles, bien que les routes soient nombreuses et en bon état de viabilité. Les villes sont le plus généralement situées sur des hauteurs, les habitations étant disposées en amphithéâtres dans le haut des versants, à l'abri des vents dominants, sans doute aussi comme résultat de l'insécurité et de la malaria dans les plaines.

L'origine de cette civilisation agraire doit être recherchée dans des temps assez lointains, car la Sicile a été, au cours des âges, soumise à de multiples influences du fait même de sa situation en Méditerranée, au confluent d'importantes lignes de communications maritimes. La colonisation Phénicienne, puis Grecque, l'occupation de Carthage, la Paix Romaine, les invasions Normandes et Arabes, le régime espagnol, puis celui des Bourbons avec le Royaume des Deux Siciles, ont successivement façonné le paysage de l'île et sa structure agraire et sociale. Sur la côte, des villes comme Palerme, Catane, Siracuse, Agrigente, portent témoignage, par leurs ruines et leurs anciens monuments, de la prospérité des vastes métropoles qui se sont succédé sur les mêmes emplacements et qui, en dehors de leur rôle de ports en relation avec tout le monde méditerranéen, devaient servir de places de commerce pour un arrière pays consacré, depuis très longtemps sans doute, à l'élevage du mouton et à la culture du blé. 
Dans les villes de l'intérieur, les monuments les plus importants: églises de style baroque et anciens palais, datent de l'occupation espagnole. Il semble qu'à cette époque il y ait eu une certaine vie urbaine autour des grands seigneurs qui possédaient de très vastes domaines cultivés d'une façon assez extensive: blé et parcours de moutons, avec l'aide d'une main-d'œuvre rurale qui résidait, sans doute déjà, de préférence dans les villes. Cette structure sociale s'explique du fait qu'il s'agissait justement d'une agriculture extensive ne demandant pas de soins constants et attentifs. La population agricole s'attachait d'autant moins à la terre, d'ailleurs, qu'elle n'était pas effectivement propriétaire du sol. Les habitations situées dans les campagnes étaient selon toute vraisemblance, des abris de pasteurs qui n'ont pas laissé de trace.

Là civilisation urbaine avec ses gens de cour, d'église et de robe, ses commerçants et ses artisans, est peu à peu entrée en décadence au fur et à mesure de l'évolution politique et de l'amélioration des communications à l'intérieur de l'île. Après le départ des Notables vers les métropoles de la côte ou le Continent, les petites villes de l'intérieur n'ont plus compté qu'une population agricole qui, en énorme extension démographique, a occupé les anciens palais et les maisons bourgeoises. La structure agraire n'ayant, par contre, subi que peu de modifications, les agriculteurs ont continué leurs anciens modes de culture, en pratiquant surtout une économie de subsistance, ce qui limitait par conséquent les possibilités de développement du commerce et de l'artisanat. Ainsi s'est façonné ce faciès de grandes agglomérations essentiellement rurales qui n'ont d'urbain que leur aspect extérieur avec leurs rues étroites et leurs places.

Sur la côte, en dehors des grandes villes qui restent en contact avec les marchés extérieurs et qui sont les métropoles du négoce, l'habitat est beaucoup plus disséminé par le fait même que depuis certainement très longtemps, la terre est consacrée à des cultures intensives : vigne, oranger, amandier, coton, riz, primeurs, etc..., qui exigent une main-d'œuvre abondante et la présence constante des agriculteurs pour les travaux renouvelés du sol et pour la surveillance des récoltes. Le coton et le riz auraient été introduits par les Arabes.

Pour obtenir le plein emploi de la main-d'œuvre agricole pléthorique et tendre à une amélioration du niveau de vie d'une population rurale dans l'ensemble pauvre, un effort considérable est actuellement en cours pour étendre les zones irriguées permettant une utilisation intensive du sol sous le climat chaud et ensoleillé de la Sicile et pour mettre en valeur les anciens grands domaines de cultures extensives avec répartition, dans ce dernier cas, des terres entre les agriculteurs.

Les bases juridiques de l'action entreprise sont essentiellement: 
- la loi forestière de I923 qui est notamment appliquée dans ses dispositions relatives à la correction des torrents et à la lutte contre l'érosion,

- la loi sur la bonification intégrale de I933, qui est à l'origine de la mise en valeur des marais Pontins, de la Maremma, de la Sardaigne (Domaine d'Arborea), etc...,

- la loi de réforme foncière de I947 permet le partage des terres entre les anciens tenanciers de grands domaines et facilite la constitution de petites exploitations agricoles,

- la loi no 646 du Io août I950 établissant une Caisse pour l'exécution des travaux spéciaux d'intérêt public dans l'Italie méridionale (Caisse du Midi) en raison du sous-développement de l'économie de l'Italie méridionale et des Iles,

- la loi de mise en valeur de la montagne du 25 juillet 1952 qui confie au service forestier toutes les tâches relatives à l'amélioration des conditions de vie des populations de montagne.

A ces textes de base, s'ajoutent les possibilités offertes par une très ancienne forme d'association de propriétaires fonciers: les Consortiums dont la constitution peut être libre ou forcée, et dont la structure rappelle beaucoup les associations syndicales françaises.

Ces différentes lois sont mises en application d'une façon extrêmement souple et permettent, grâce aux possibilités d'articulation qui existent entre elles, de disposer d'importants crédits et de moyens judiriques puissants adlaptés aux conditions particulières du milieu.

Pour fixer les idées sur les possibilités d'action ainsi offertes aux services publics, il nous a été permis d'examiner l'exemple de la zone de bonification des fleuves Platani-Tumarno.

La Caisse du Midi s'est vu confier la tâche essentielle d'établir un plan général de remise en valeur de la Sicile, suivant des modalités voisines de celles offertes en France par les lois de programme. De gros moyens financiers sont à cet effet mis à sa disposition. Elle a la responsabilité directe de la réalisation de la réforme agraire proprement dite (loi de 1947) et a chargé l'Office de Réforme Agraire Sicilien (ente reforma agraria siciliana E.R.A.S.) du soin de dresser et d'exécuter le programme de bonification intégrale des terres (loi de I933) dans la zone de bonification (PlataniTumarno) suivant les données approuvées au préalable par décret pris en Conseil des Ministres à Rome. L'E.R.A.S. joue un rôle comparable en France à celui d'une Société d'économie mixte telle. que la Compagnie Nationale d'Aménagement de la région du BasRhône et du Languedoc dont la mission est également définie par décret. L'E.R.A.S. à son tour, a confié les travaux d'amélioration foncière, à un Consortium, de la même manière qu'en France, 1'Administration du Génie rural est appelée à grouper les propriétaires 
pour certains travaux de drainage ou d'irrigation. Dans le cadre du plan réalisé par la 'Caisse du Midi et l'Office de Réforme Agraire Sicilien, le service forestier a la responsabilité directe de certains travaux financés par la loi forestière de I923, correction de torrents, ou la loi sur la montagne et dont l'exécution a été confiée à une société privée: entreprise de reconstitution de la montagne: I.R.M.O.

Ce schéma peut d'ailleurs être modifié suivant les circonstances, et la Caisse du Midi, dans certains cas, ne passera pas par l'intermédiaire d'un Office de Réforme Agraire et confiera les travaux directement à un Consortium.

Il est nécessaire de souligner la souplesse de ces dispositions qui, en fonction de chaque situation particulière, sont adaptées à la nature des travaux, à la structure sociale et, enfin, aux disponibilités financières rattachées aux différentes lois mises en application.

Le voyage d'études a permis de prendre connaissance d'un certain nombre de travaux qui peuvent être classés en deux grandes catégories:

$I^{n}$ les travaux ayant pour but d'étendre, grâce à l'irrigation essentiellement, les possibilités de culture intensive dans les terrains des vallées des fleuves et de la région côtière,

$2^{\circ}$ les travaux ayant pour objet d'améliorer les conditions de vie dans les zones restant consacrées, notamment au centre de l'île, à une culture extensive, par suite des conditions de sol et de climat.

Parmi les travaux de la première catégorie, on peut citer la substifution de cultures irriguées d'orangers sur terrasses, à la vigne et à l'olivier, ou même à des cultures extensives de blé, aux environs de Caltagirone, l'extension de la culture du coton dans la même région, le développement des orangeraies sur la bande côtière entre Messine et Catane.

Aux mêmes préoccupations se rattache la mise en valeur de la vallée de la Gela, grâce à la construction d'un barrage dans la partie haute du fleuve, dont le bassin d'alimentation a été reboisé par le service forestier pour éviter les causes d'atterrissement du lac de retenue qui peut contenir I4 millions de mètres cubes d'eau. Dans la vallée de la Gela, la propriété est déjà assez morcelée et l'irrigation doit permettre de remplacer, sur plus de 6000 hectares, la culture classique: blé, suivie de pâturage de moutons, par des cultures plus rémunératrices, telles que le coton, les artichauts, les tomates, etc... Les services publics s'efforcent de convaincre les agriculteurs de poursuivre cette reconversion et, à cet effet, une exploitation pilote a été créée par les services agricoles. Les agriculteurs ne sont pas forcés de prendre l'eau d'irrigation, et ceux qui l'utilisent paient june redevance minime. Il est certain qu'actuellement beaucoup 
d'agriculteurs restent fidèles aux anciennes traditions, mais avec le temps, une évolution se fera certainement sentir.

On peut noter également le développement des cultures maraîchères de la région d'Agrigente.

Dans la région de Taormine, le tourisme a apporté des ressources importantes aux citadins-agriculteurs qui conservent en culture un certain nombre de champs en terrasses pour la satisfaction de leurs propres besoins domestiques. Ainsi, grâce à l'appoint de ressources extérieures, une agriculture peu évoluée se maintient.

Dans l'ensemble, ces travaux de bonification, en permettant une utilisation plus intensive du sol, sont certainement rentables aussi bien au point de vue financier qu'économique et social. La seule préoccupation des pouvoirs publics doit être, en fonction de la création d'une économie de marché, de rechercher pour les produits de haute valeur ainsi cultivés, des débouchés possibles, soit vers les grandes villes de Sicile, soit vers le Continent. Cet aspect de la question n'est pas à négliger. La culture du coton doit déjà faire l'objet d'une protection en raison de son prix de revient supérieur aux cours mondiaux. L'extension de la culture de l'oranger, qui n'est pas propre à la Sicile ou à l'Italie, peut amener une surproduction dont il sera nécessaire de prévoir l'écoulement.

Les travaux de mise en valeur des zones de culture extensive, qui intéressent le centre de la Sicile, posent pourtant des problèmes beaucoup plus graves, notamment au service forestier. Celui-ci a toutefois une action limitée car, pratiquement, sauf en haute altitude, toutes les terres sont consacrées à la culture, les champs montant jusqu'aux sommets des versants.

Au cours du voyage, on peut dire qu'il n'a jamais été rencontré de friches ou de maquis. Les forêts naturelles dont quelques lambeaux ont été observés près de Cammarata n'occupent d'ailleurs qu'une surface extrêmement faible: $2 \%$ du territoire. Le paysage du centre de l'île est donc très caractéristique avec son absence presque totale d'arbres, sauf quelques vergers: amandiers, oliviers ou caroubiers.

Dans les moins bons sol, en raison de la sécheresse de l'été, les cultures souvent avec jachères, n'ont qu'un faible rendement et, dans certaines régions, provoquent des phénomènes d'érosion très graves,

Finalement, " la soif de la terre » qui sévit en Sicile, oblige le service forestier à avoir une action limitée et prudente, qui doit trouver sa justification à un triple point de vue:

- assurer une défense des sols,

- donner du travail à la main-d'œuvre locale,

- assurer le ravitaillement en matières premières des usines.

En ce qui concerne la défense du sol, le territoire de la Sicile comporte, en gros, $2 / 3$ de terres sablo-argileuses dont certaines sont 
rendues instables par les abus de jouissance, et $\mathrm{I} / 3$ de terrains calcaires qui prennent parfois l'aspect désertique du Karst. Dans les terrains sablo-argileux, tels que ceux qui ont été visités près de Caltagirone, le service forestier achète, puis reboise les terres en glissement de façon à assurer la protection des terres de culture et, dans le cas particulier, le maintien des assises même de l'agglomération. Il s'agit le plus souvent, de couches de sable pliocèniques qui alternent avec des couches d'argile. L'essence employée est l'eucalyptus.

Aux abords de Modica, en zone calcaire, des banquettes ont été aménagées 'sur des versants abrupts; une autre réalisation a été observée à Cammarata où le reboisement d'anciens parcours a été poursuivi sur I 500 hectares. Dans ce dernier cas, le pin d'Alep est employé à basse altitude et les feuillus, notamment les chênes, à haute altitude.

Enfin, le service forestier a la responsabilité de la fixation des dunes côtières, dans la région de 'Gela, par exemple.

Le second aspect du reboisement, source de travail, a été notamment noté dans la région de Modica où des banquettes en terrain difficile ont pu être aménagées dans des conditions de rentabilité acceptables du fait même que les travaux ont été exécutés par les propriétaires eux-mêmes, à proximité d'une ville, pour la protection et le ravitaillement de cette ville.

Le troisième aspect des boisements est représenté par un projet de plantation en eucalyptus portant sur 12 ooo hectares et qui a été envisagé dans la région d'Enna et de Piazza Armerina. Pour sa réalisation, un accord a été passé entre le Gouvernement de la région sicilienne et le Groupe industriel de la Snia Viscosa. Le but du boisement qui devrait porter sur les plus mauvais terrains et les versants, en maintenant les cultures et les vergers sur les stations les plus favorables, serait d'assurer par des taillis d'euvalyptus traités à courte rotation, la matière première nécessaire à une industrie de la cellulose à établir dans la région.

La Société SNia VIscosa, par l'intermédiaire d'une Société fiiliale, la Siage, achète des terres et les reboise en faisant appel à une Société, l'Irmo, et, en bénéficiant de l'aide de la Caisse du Midi; elle se constitue ainsi une ressource pour ses usines. Le Gouvernement de la région procède lui-même directement aux boisements, ou par l'intermédiaire de l'Irmo, en bénéficiant également des prêts prévus par la Caisse du Midi. Le programme progresse de façon satisfaisante mais lentement, car il importe de ne pas retirer des terres aux agriculteurs.

Sur un plan plus général, on se trouve dans la partie centrale de l'île, devant une population agricole extrêmement dense pratiquant une culture très extensive et où l'exode rural ne semble absolument 
pas amorcé, contrairement à ce qui se passe dans certaines régions de l'Italie centrale.

Devant de telles difficultés, il est certain que les impératifs de la rentabilité financière des investissements pour la mise en valeur de cette région s'effacent devant les nécessités sociales. Sans doute on doit espérer que la multiplication des zones de culture intensive permettra de desserrer progressivement l'étreinte d'une population agricole sur des terres à faible rentabilité, mais dans l'immédiat, il importe avant tout, de donner une occupation à la main-d'œuvre agricole disponible. En principe, dans les régions du centre de l'île, les grands domaines ont été morcelés en petites propriétés de 5 à ro ha isur des sols pauvres. Il est à craindre que le revenu de telles propriétés soit insuffisant pour assurer, à lui seul, la vie de ceux qui cultivent la terre.

Au cours de ces dernières années, la politique suivie était de disséminer l'habitat dans les zones du centre de l'île. Il semble que la population qui est très attachée à ses traditions anciennes, quitte difficilement les villes pour aller s'installer dans des fermes isolées. On peut d'ailleurs se demander si, dans les conditions économiques où vivent ces populations, les charges publiques d'un habitat disséminé (infrastructure routière, services publics et sociaux) pourront être aisément supportées. On revient d'ailleurs, actuellement à la notion de petites agglomérations avec école, mairie, où il sera possible d'avoir une certaine vie de communauté.

La nécessité d'augmenter le revenu des petites propriétés ne doit pas faire perdre de vue l'impératif de la conservation du « patrimoine-sol ). Dans l'état actuel ide l'agriculture sans apport d'engrais massifs, les labours profonds pourraient augmenter la production traditionnelle du blé, mais risqueraient à longue échéance, d'entraîner une dégradation du sol qui, finalement, pour des gains immédiats auraient sur l'avenir de très graves répercussions. Dans une île où toutes les terres sont nécessaires pour assurer la vie d'une population, la conservation du sol est essentielle et c'est à cet égard que le service forestier joue le rôle le plus important.

Il ne semble pas, compte tenu de la structure sociale, que d'autres programmes que ceux définis par la Caisse du Midi, tout au moins, lors de la création de celle-ci en I950, aient mérité d'être retenus. Il s'agissait, avant tout, de faire vivre une population.

La politique forestière en Sicile a été, au cours de cette période de 1950-1956, étroitement liée aux progrès de l'agriculture et le service forestier a joué un rôle important dans une certaine remise en ordre de la vie rurale en Sicile. Il a à son actif des réalisations remarquables. Son action s'est trouvée néanmoins limitée par le fait que l'extension de l'état boisé a été constamment freinée par la pression démographique des populations rurales, notamment au centre de l'île. 
Dans l'immédiat, en dehors des boisements d'essences à croissance rapide de la région Enna-Piazza-Armerina, dont il a été parlé cidessus et qui sont, à certains égards, permis par la bonification du fleuve Simeto, on doit surtout s'attacher à des problèmes de restauration et de conservation des sols et on ne doit pas hésiter dans ce cas à faire appel à d'autres cultures, telles que, par exemple, les arbres fruitiers sur terrasses, chaque fois que la chose est possible.

Finalement, tant que de larges débouchés n'auront pas été trouvés pour la main-d'œuvre agricole excédentaire, la situation restera sérieuse et l'avenir d'autant plus lourd de difficultés que la pression continue à s'accroitre. Sans doute les travaux de bonification portant sur des cultures intensives doivent créer des " Pôles de croissance ", suivant l'expression du Professeur Perroux, en développant dans certaines cités rurales, des activités commerciales et artisanales, amorces d'une vie économique plus intense, et sources de nouveaux emplois.

Mais, en fait, se trouve maintenant posé le problème de l'industrialisation de la Sicile, rendu plus actuel par la découverte des gisements de pétrole de Siracuse.

Des exemples récents montrent qu'un développement trop exclusif de l'industrie lourde sans que le progrès de l'agriculture ait suivi la même cadence amène des distorsions économiques graves. De même, une agriculture évoluée sans un effort industriel parallèle ne règle pas le problème du plein emploi de la main-d'œuvre et n'apporte ainsi que des solutions partielles et provisoires.

C'est la raison pour laquelle un projet de loi a été déposé par le Gouvernement italien pour proroger et amplifier l'action de la Caisse du Midi. L'activité de la Caisse qui devait prendre fin en I960 se poursuivra jusqu'en 1965. Le Budget annuel qui est de Ioo milliards de lires, passera à I 30 milliards au cours de l'exercice financier I959-I960, et à I 50 milliards au cours des exercices I960-I96I et ultérieurs. Les prévisions de crédit sont au total de I 870 milliards.

L'innovation la plus importante est que la Caisse du Midi qui, dans son organisation primitive ne finançait que des travaux publics ou de bonification agraire, pourra désormais intervenir dans le secteur industriel par le biais, notamment, de subventions d'équipement et de bonifications d'intérêts. La Caisse du Midi est habilitée pour accorder aux industries, petites et moyennes, s'établissant dans des localités de moins de 75000 habitants, une subvention couvrant jusqu'à $20 \%$ du coût des installations fixes. Elle contribuera pour $50 \%$ aux avances d'aménagement par les collectivités locales des zones industrielles dont la création est, par ailleurs, facilitée sur le plan juridique. Elle accordera aux Instituts de crédit à moyen terme des bonifications d'intérêt sur leurs emprunts obligataires.

Parallèlement à cet effort consenti par la Caisse du Midi en fa- 
veur du secteur industriel, le revenu des sociétés italiennes sera exempt d'impôts à concurrence de $50 \%$ de son montant s'il est réinvesti dans le Sud.

$\mathrm{Si}$ les différentes mesures prévues dans le projet de loi sont adoptées par le Gouvernement, l'Italie disposera d'une législation en faveur de l'aménagement régional, extrêmement complète, dont nous pourrons nous inspirer avec profit.

Dans le cadre ainsi élargi d'une politique de mise en valeur et de modernisation, le Service forestier, loin de voir diminuer sa tâche, verra, au contraire, augmenter ses possibilités. Dans la mesure où les agriculteurs cesseront d'exercer une pression trop forte sur les terres, il sera possible de poursuivre et d'amplifier les travaux de reboisement, soit pour la conservation des sols, soit pour l'augmentation des ressources forestières en vue de la création d'une industrie d'utilisation du bois amorcée en matière d'eucalyptus par la SNIA Viscosa.

René G. Fontaine. - Jean de Vaissière. 